\title{
Research on the Temperature Characteristics of Movable Tooth Trans- mission Motor of Electromagnetic Harmonic
}

\author{
Ren Yubo* and Xu Lizhong
}

Parallel Robot and Electromechanical System Laboratory, Yanshan University, Qinghuangdao, Hebei, 066004, China

\begin{abstract}
Based on the heat transfer characteristics of electromagnetic harmonic movable tooth transmission motor, this paper adopted the finite element method to establish a three-dimensional temperature field model for numerical calculation on the basis of four basic assumptions. It analyzed the heat loss and gave out the functional to calculate the temperature field within each medium and obtain the temperature distributing curves. Then taking the prototype as an example, it calculated the steady-state temperature field of the system under different loading. The comparison between the results and measured values has verified the reasonableness of the established model and calculation method employed. The results could provide a theoretical basis for the optimized design of electromagnetic harmonic tooth transmission system.
\end{abstract}

Keywords: Electromagnetic harmonic, loss, numerical calculation, temperature field.

\section{INTRODUCTION}

As electronic and control technology continues to penetrate the mechanical field, the generalized complex mechanical transmission has become a leading international subject in mechanical fields [1-4] for this concept is anti-tradition and could achieve the organic combination of mechanics, electricity and control. The electromagnetic harmonic movable tooth transmission system involved in this paper is a kind of organic combination of harmonic transmission technology, electromagnetic transmission technology, movable tooth transmission technology and control technology, that is, a new electromechanical integrated complex transmission system [5-6].

The electromagnetic harmonic movable tooth transmission motor is an electromagnetic -mechanical energy conversion device. Due to its long-term continuous operation, the energy loss is eventually converted to heat, so that the temperature of each part of the motor increases which has a direct impact on the life of all the insulating materials. Meanwhile, the viscosity of lubricating oil of the may decrease, which may damage the lubrication state between the movable tooth, center wheel and tooth holder, and ultimately limit the output of the system. And the electromagnetic harmonic transmission is operating in low speed, its running state corresponds to the operating state of starting or braking and the efficiency distribution has a great influence on the electromagnetic and its design and structure. Therefore, the study of loss and temperature distribution of electromagnetic harmonic movable tooth transmission is of great significance for the analysis of its failure mechanisms, the improvement of its operating performance and effective control of its lubricating and cooling. The experts and scholars have conducted a large number of studies on the motor temperature field, for example, make use of the general collection model to analyze the magnetic fields, temperature field, vibration characteristics in the motor, and give the inter-coupling methods of each field [7]; use the T-type equivalent lumped model and the traditional thermal resistant circuit to solve the motor temperature field [8]; use the lumped parameter and thermal network diagram to conduct coupled solution for the temperature field and electromagnetic field [9-10]; utilize the optimized heat transfer method of high torque density motor to carry out loss calculations and determine the heat loss coefficient [11-13].

Based on the heat transfer characteristics of electromagnetic harmonic movable tooth transmission motor, this paper adopted the finite element method to study its heat transfer and temperature rise characteristics under different loading. Then use the prototype test to verify the theoretical analysis and provide a theoretical basis for the study of harmonic motor temperature field.

\section{NUMERICAL CALCULATION OF THE TEMPER- ATURE FIELD OF ELECTROMAGNETIC HARMON- IC MOVABLE TOOTH TRANSMISSION MOTOR}

\subsection{Establishment of the Solving Domain Model}

Taking the prototype as an example, this paper established a three-dimensional temperature field model of the motor. As the electromagnetic harmonic movable tooth transmission motor has the core-coil, movable tooth, and tooth rack and center wheel in the axial direction, take the solving domains of two temperature fields at the location of core-coil and movable tooth. The basic parameters of the system are as shown in Table $\mathbf{1}$ and the physical model is as shown in Fig. (1). 
Table 1. Harmonic electromagnetic movable teeth transmission motor parameters.

\begin{tabular}{|c|c|c|c|}
\hline Item & Value & Item & Value \\
\hline Rated power $P_{e}$ & $100 \mathrm{~W}$ & Rated voltage $U_{e}$ & $220 \mathrm{~V}$ \\
\hline Number of pole pairs $p$ & 1 & Stator slots $m$ & 24 \\
\hline Number of turns per slot $N_{Z}$ & 81 & Rated current $I$ & $0.56 \mathrm{~A}$ \\
\hline Outer diameter of stator $\mathrm{D}(\mathrm{mm})$ & 118 & Inner diameter of stator $d(\mathrm{~mm})$ & 68 \\
\hline Length of iron core $l_{\mathrm{T}}(\mathrm{mm})$ & 63 & Flexspline length $l_{(\mathrm{mm})}$ & 127 \\
\hline Outer diameter of flexspline $\mathrm{r}(\mathrm{mm})$ & 67 & Flexspline thickness $\mathrm{t}(\mathrm{mm})$ & 0.2 \\
\hline air gap $\delta(\mathrm{mm})$ & 0.5 & Core material of stator & $35 \mathrm{WW} 270$ \\
\hline Phase Resistance ${ }_{R}(\Omega)$ & 0.188 & Phase inductance $L$ & $0.234 \mathrm{mH}$ \\
\hline
\end{tabular}

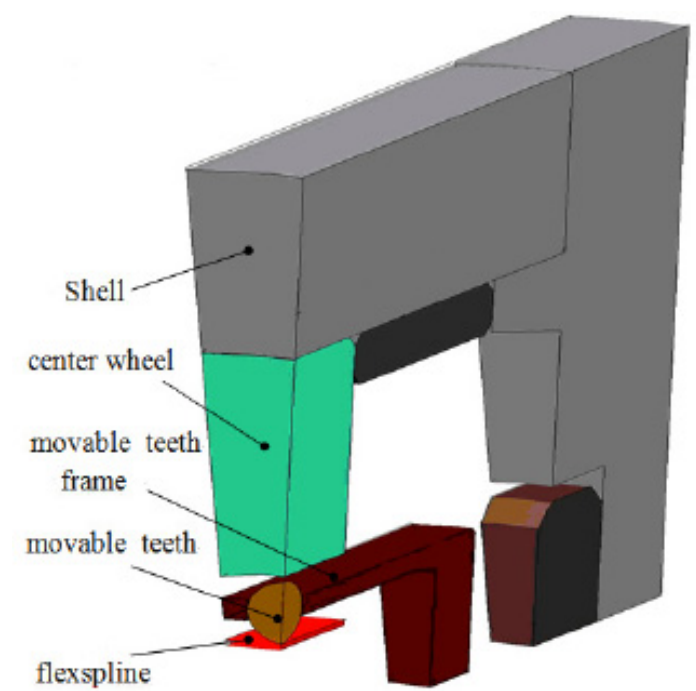

Fig. (1). Temperature field solving regional.

Based on the characteristics of electromagnetic harmonic movable tooth transmission system, this paper made the following assumptions:

(1) In the system, the heat transfers by the heat conduction; on the surface of the system, the heat dissipates by the natural heat convection. Due to the low rotating speed of the system, the axial flow of the air gap between the stator and flexspline is relatively small, so it is considered that the heat flow between the stator and the flexible wheel could generate heat exchange by the air conduction.

(2) Assuming that all of the copper and iron losses are generated in the effective length at the axial direction and the cooling on the surface of the system is also converted within this range, then they can be modeled as a twodimensional field problem. As there are no high-speed rotating components and no effects of the fan in the system, so that no axial heat-flux caused by forced convection is found within the effective area of the stator and the flexible shaft. This indicates that two-dimensional

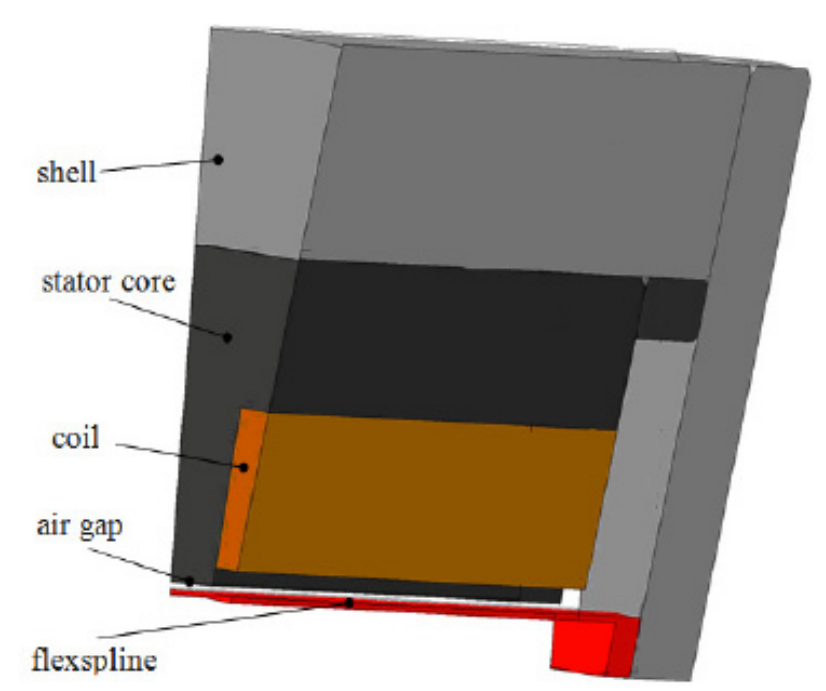

model is suitable for the characteristics of electromagnetic harmonic tooth transmission.

(3) Calculate the steady-state temperature field. Since the current of the transmission of electromagnetic harmonic tooth is almost unchanged, it follows that copper loss is constant.

(4) According to the symmetry, seek solution based on the range of $1 / 2$ stator tooth as the object.

\subsection{Heat Source Within the System}

Electromagnetic harmonic tooth transmission is an electromagnetic - mechanical energy conversion device. Energy loss in action is eventually converted to heat energy and it finally reaches steady state equilibrium by the heat exchange between the motor components with the environment. Accurately calculating the losses in the motor system is a prerequisite for the analysis of temperature field. The loss of the electromagnetic harmonic tooth transmission is comprised of electromagnetic loss and mechanical loss. 


\subsubsection{Electromagnetic Loss}

Part of system input electric power is consumed in the stator resistance, producing the electron copper loss, while the other part of the consumption is in the resistance field, resulting in the stator iron loss. Besides, the additional loss including flexspline eddy current loss and hysteresis loss should also be considered. The remaining power is applied to flexspline through the air gap magnetic field- $P_{M}$.

\subsubsection{Electronic Copper Loss}

In the harmonic electromagnetic tooth transmission process, the alternating electric current flows through a conductor which will lead to the skin effect and proximity effect in the surrounding magnetic field [14]. The additional losses caused by them are defined as eddy-current loss, while the eddy-current loss and DC loss are collectively known as AC loss (copper loss) [15], namely

$$
P_{c u}=P_{z}+P_{w}
$$

$P_{c u}$ is the AC loss; $P_{z}$ is the DC loss; $P_{z}=I^{2} R_{z} ;{ }^{w}$ is eddy-current loss; $I$ is the virtual value of current; $R_{z}$ is DC resistance.

Assuming that the magnetic field in the harmonic motor slot is parallel to its bottom, ignore the effects of eddy current of the conductor on the magnetic field and the eddycurrent loss of the conductor is shown as follows [16].

$$
P_{w}=\frac{\pi \omega^{2} B^{2}{ }_{\alpha} d^{4}}{128 \rho_{c}}
$$

$d$ is the diameter of the conductor; $l_{d}$ is the length of the conductor; $\rho_{c}$ is the resistivity of the conductor; $B$ is the amplitude of flux density; $\omega$ is the frequency of flux density angular.

\subsubsection{Iron Core Loss}

The stator core loss is one of the main losses of harmonic electromagnetic tooth transmission system and accounts for a large proportion of the total losses. It is usually refers to the sum of tooth iron loss and iron loss of the yoke part.

$$
P_{F e}=P_{c}+P_{\alpha}
$$

and

$$
\left\{\begin{array}{l}
P_{c}=k_{z} p_{0} B_{c}^{2} f^{13} G_{c} \\
P_{\alpha}=k_{\alpha} p_{0} B_{\alpha}^{2} f^{13} G_{\alpha}
\end{array}\right.
$$

$k_{z}, k_{a}$ is the coefficient with additional loss; $p_{0}$ is the specific losses of core material; $B_{c}$ is the tooth flux density; $B_{a}$ is the yoke flux density; $f$ means the frequency; $G_{c}$ means the tooth weight; $G_{a}$ means the yoke weight.

\subsubsection{Mechanical Power Loss}

(1) The power loss of engagement pair

The analysis indicates that there is relative rolling motion between the movable tooth and flexible wheel, there is rela- tive sliding between the movable tooth and the tooth carrier, and there are both relative rolling and relative sliding between the tooth and the center wheel. $P_{1}^{i}, P_{2}^{i}, P_{3}^{i}$ respectively refers to the power loss when the $i$ th movable tooth contacts with the flexible wheel, the tooth carrier and the center wheel. The meshing power loss of a single tooth is

$$
\begin{aligned}
& P_{f}^{i}=P_{1}^{i}+P_{2}^{i}+P_{3}^{i} \\
& P_{1}^{i}=\omega_{1 i} F_{1 i f}^{\prime} f_{1 i} \\
& P_{2}^{i}=V_{2 i} F_{2 i f}^{\prime} f_{2 i} \\
& P_{3}^{i}=\omega_{3 i} F_{3 i f}^{\prime} f_{3 i}
\end{aligned}
$$

in the formula, $F_{1 i f}^{\prime}, F_{2 i f}^{\prime}, F_{3 i f}^{\prime}$ means the friction between the movable tooth and the flexible wheel, tooth rack and center wheel; $f_{1 i}$ and $f_{2 i}$ is the friction coefficient of the movable tooth with the flexible wheel and tooth rack; $\omega_{1 i}$ means the relative rolling angular velocity between the movable tooth and the flexible wheel; $V_{2 i}$ means the relative sliding angular velocity between the movable tooth and the tooth rack; $\omega_{3 i}$ means the relative rolling-sliding angular velocity; $f_{3 i}$ means the rolling-sliding friction coefficient between the movable tooth and the flexible wheel.

The electromagnetic harmonic movable tooth transmission has multi-tooth engagement simultaneously; at any time, the friction power loss on each movable tooth is not the same, coupled with the input power, which is because there are always half of the movable teth in two regions of engagement operating, so the power loss at any moment is shown as follows:

$$
P_{f}=\sum_{i=m}^{n} P_{f}^{i}+\sum_{i=p}^{q} P_{f}^{i}
$$

${ }^{m}, n$ and $p, q$ respectively represents the number of movable tooth in two different regions of engagement.

(2) The power loss of the bearing

The power loss of a single bearing

$$
P_{z c}=\pi n_{z} M_{f} / 30
$$

$n_{z}$ is the rotation speed of the inner ring of the bearing; $M_{f}$ is the friction torque of the bearing.

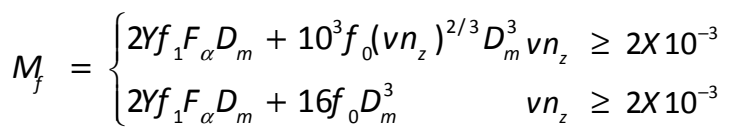

$Y$ and ${ }^{H_{a}}$ respectively represents the axial coefficient and axial load of the bearing; $V$ is the kinematic viscosity of the lubricant; ${ }_{m}$ is the average diameter of the bearing; ${ }_{0}$ and $f_{1}$ are the coefficients related to the bearing type and lubrication methods.

\section{(3) Power loss due to oil agitation}

The power loss due to oil agitation mainly includes that of flexspline and movable tooth. For the spherical surface (radius of $r^{\prime}$ and the effective area $A$ ) rotating at the angular velocity of $\omega^{\prime}$ in the viscous fluid medium (density $\rho^{\prime}$, kin- 
ematic viscosity $v$ and friction coefficient $f^{\prime}$ ), the power loss due to oil agitation is shown as follows:

$$
P_{y}=\frac{1}{8} \rho f^{\prime} A \omega^{\prime 3} \gamma^{\prime 3}
$$

In the above formula

$$
f^{\prime}= \begin{cases}16 / R_{e} & T_{\alpha}<41 \\ 3\left(R_{e} / 2500\right)^{0.856}\left(16 / R_{e}\right) & R_{e} \geq 2500 \\ 1.3\left(T_{\alpha} / 41\right)^{0.539}\left(16 / R_{e}\right) & T_{\alpha} \geq 41\end{cases}
$$

The Reynolds number $R_{e}=\omega^{\prime} r C / v$, the Taylor number $T_{a}=R_{e} \sqrt{C / r^{\prime}}, C$ is the characteristic gap surrounding the ball surface. lows

The total losses of mechanical power is shown as fol-

$$
P=P_{f}+P_{z}+P_{y}
$$

\section{(4) Rolling-sliding friction coefficient}

Based on the previous analysis, there are both rolling and sliding when the tooth contact with the center wheel, so the corresponding friction is referred to as rolling-sliding friction. The work done by the rolling friction, sliding friction and rolling-sliding friction is respectively shown as follows

$$
\begin{aligned}
& w_{1}=\mu_{1} F_{3 i} S_{1} \\
& w_{2}=\mu_{2} F_{3 i} S_{2} \\
& w_{3}=\mu_{3} F_{3 i} S_{3}
\end{aligned}
$$

${ }^{\mu_{1}}$ refers to the rolling friction coefficient; $\mu_{2}$ is the sliding friction coefficient; $f_{3 i}$ is the rolling-sliding friction coefficient; $F_{3 i}$ is the positive pressure imposed on the movable tooth by the center wheel; $S_{1}$ refers to the arc length of engagement of movable tooth, that is, the rolling distance; $S_{2}$ refers to the sliding distance; ${ }^{S_{3}}$ refers to the arc length of engagement of center wheel, that is, the rolling-sliding distance.

The work done by the rolling-sliding friction is equal to the sum of the work done by rolling friction and sliding friction; therefore

$f_{3 i} S_{3}=\mu_{1} S_{1}+\mu_{2} S_{2}$ mula

$S_{1}$ can be approximately calculated by the following for-

$S_{1} \approx r_{b} \varphi_{v}$

$r_{b}$ is the radius of the movable tooth; $\varphi_{\nu}$ is the wrap angle of the centered tooth; $z_{z}$ is the wave number of the centered tooth.

$S_{3}=\left(r+2 r_{b}\right) \varphi_{2}$

$r$ is the radius of flexspline.

$\mathrm{S}_{3}=\mathrm{S}_{1}+\mathrm{S}_{2}$
Finally

$f_{3 i}=\frac{\left[\left(\mu_{1}-\mu_{2}\right) r_{b} \varphi_{v}+\mu_{2} \varphi_{2}\left(r_{b}+2 r_{b}\right)\right]}{\left(r_{b}+2 r_{b}\right) \varphi_{2}}$

\subsection{Cooling Conditions for the Motor of Electromagnetic Harmonic Tooth Transmission}

Heat loss generated within the system of electromagnetic harmonic tooth transmission must be disseminated through a certain way. There are three modes of heat transferring- conduction, radiation and convection, of which the heat source is to rely on conduction when it is transferred to the surface in contact with air, while it is through convection and radiation when it is spread into the air from the surface, mainly relying on convection.

Fourier's law states that [17] the heat within the object is transferred from the hot zone to the low temperature zone, with heat flux per unit area proportional to the temperature gradient, that is to say,

$$
Q=-\lambda S \frac{\partial T}{\partial n}
$$

In the formula, $Q$ stands for the heat flux, $\lambda$ refers to the thermal conductivity, $S$ is the heat transfer area, $\partial T$ represents the temperature gradient, and the negative sigm indicates that heat is conducted from the hot temperature zone to the low temperature zone.

At the junction of solids and gases, heat convection occurs as the flow of gas takes the heat away. Heat convection effect is determined by Newton's law of cooling.

$Q=\alpha S\left(T_{W}-T_{A}\right)$

In the formula, $\alpha$ refers to the thermal convection coefficient, $T_{W}$ stands for the temperature on the surface of objects, and $T_{A}$ is the temperature of the gas.

Besides, thermal energy will produce thermal radiation in the form of electromagnetic waves. As for black body, Stefan-Boltzmann law states that the radiation flux is proportional to the fourth power of the absolute temperature, that is to say,

$Q=\sigma S\left(T_{w}^{4}-T_{s}^{4}\right)$

In the formula, $\sigma$ is the Stefan-Boltzmann constant, whose value is $5.669 \times 10^{-8} \mathrm{~W} / \mathrm{m}^{2} \mathrm{~K}^{4}$, and $T_{S}$ refers to the absolute temperature of the surrounding environment.

The above formula applies only to the thermal radiation of blackbody. For general metal surfaces, the heat radiated is not as much as blackbody, but the total radiated heat is still proportional to $T^{4}$.

As electromagnetic tooth harmonic transmission system has no high-speed rotating parts, the air flow can be considered natural convection rather than forced convection and the system heat, after transferred to the surface, is dissipated through natural ventilation, including radiation.

\subsection{Mathematical Model of Temperature Field}

As is shown in Fig. (1), the temperature field of electromagnetic harmonic tooth transmission is a field with a varie- 
ty of media. According to the Fourier heat conduction law, it can be drawn that the temperature field within each medium is shown as the following formula:

$$
\Omega: \nabla\left(\lambda_{i} \nabla T_{i}\right)=-Q_{1}
$$

In the formula, $\Omega_{i}$ is the $i^{\text {th }}$ kind of medium area, $\lambda_{i}$ stands for the thermal conductivity of the $i^{\text {th }}$ kind of medium, $T_{i}$ represents the temperature within the $i^{\text {th }}$ kind of medium, $Q_{i}$ signifies the heat generation rate per unit volume within the $i^{\text {th }}$ kind of medium.

At the interface of each medium, due to the temperature continuity and continuity conditions of heat flux meets the following condition

$$
\left\{\begin{array}{l}
T_{i}=T_{j} \\
-\lambda_{i}\left(\frac{\partial T}{\partial n}\right)_{i}=-\lambda_{j}\left(\frac{\partial T}{\partial n}\right)_{j}
\end{array}\right.
$$

In the formula, $i, j$ respectively represent the adjacent two media.

As the heat flux on the system surface is dissipated by natural heat convection, the temperature meets the boundary condition

$\left.-\lambda\left(\frac{\partial T}{\partial n}\right)_{i}=\alpha T-T_{0}\right)$

In the formula, $\lambda$ is the thermal conductivity of the housing case, $\alpha$ refers to the equivalent heat dispersion coefficient on the surface, and $T_{0}$ stands for room temperature.

Bases on the above formulas, the temperature field problems of electromagnetic harmonic tooth transmission can be unified as the following formula.

$$
\left\{\begin{array}{l}
\Omega: \nabla(\lambda \nabla T)=-Q_{1} \\
s:-\lambda\left(\frac{\partial T}{\partial n}\right)=\alpha\left(T-T_{0}\right)
\end{array}\right.
$$

In the formula, $\Omega=\sum \Omega$ is the he total area, $S$ stands for the surface of the housing case.

This is the mathematical model of the temperature field of electromagnetic harmonic tooth transmission.

Use Galerkin method to conduct weighted integral on Formula (16)

$$
\text { \#! }[(! \quad T)+Q] W d !=0
$$

It can be obtained from the integral theorem that

\#! ! W $\cdot T d !+\#^{2} Q W d !+\#_{s} ! W \frac{T}{n} d S=0$

Discretize field and take function $W=N_{i}$ as the shape function, then a finite element equation can be obtained based on the above formula

$[K]\{T\}=\{P\}$

Thermal conductivity is related to temperature. According to literature [15], we can obtain the thermal conductivity of some materials, as is shown in Table 2 below.
Table 2. Thermal conductivity of the material $(\mathrm{W} / \mathrm{m} 0 \mathrm{C})$.

\begin{tabular}{|c|c|c|}
\hline \multirow{3}{*}{ Material } & Temperature & $\begin{array}{c}\text { Thermal } \\
\text { conductivity }\end{array}$ \\
\hline \hline \multirow{3}{*}{ copper } & $20^{\circ} \mathrm{C}$ & 386 \\
\cline { 2 - 3 } & $100^{\circ} \mathrm{C}$ & 379 \\
\cline { 2 - 3 } & $200{ }^{\circ} \mathrm{C}$ & 374 \\
\hline \multirow{4}{*}{ carbon steel } & $20^{\circ} \mathrm{C}$ & 43 \\
\cline { 2 - 3 } & $100^{\circ} \mathrm{C}$ & 43 \\
\hline \multirow{7}{*}{ air } & $200^{\circ} \mathrm{C}$ & 42 \\
\cline { 2 - 3 } & $20^{\circ} \mathrm{C}$ & 0.0257 \\
\cline { 2 - 3 } & $100^{\circ} \mathrm{C}$ & 0.0317 \\
\cline { 2 - 3 } & $200^{\circ} \mathrm{C}$ & 0.0419 \\
\hline
\end{tabular}

Therefore, Formula (19) becomes the nonlinear equation of $T$, wherein the relative value of the thermal conductivity of the solid varying with the temperature is small, while that of the gas is more significant. The thermal conductivity of the material at the temperature of $T$ is

$\lambda=\lambda_{0}\left[1+\beta\left(T-T_{0}\right)\right]$

In the formula, $\lambda_{0}$ is the thermal conductivity at the temperature of ${ }^{T_{0}}, \beta$ refers to the temperature coefficient of the thermal conductivity.

So according to Newton-Raphson method, expand Formula (19) at $(T)^{(n)}$, and we can get the iterative formula

$J\left(\{T\}^{(n+1)}-\{T\}^{(n)}\right)=\{P\}^{(n+1)}-\{P\}^{(n)}$

In the formula, $J$ stands for Jacobi matrix, and $n$ represents the $n^{\text {th }}$ iteration. When the temperature of two iterations is less than a given value, it is believed that the result of the $n^{t h}$ iteration is the required temperature.

\section{RESULTS AND ANALYSIS}

Based on the numerical calculations of the temperature field, the temperature distribution curve of the prototype is as shown in Fig (2) and it can be seen from the figure:

(1) As the electromagnetic harmonic motor operates in the braking state, the temperature of flexspline is relatively high; when the frequency is $36 \mathrm{~Hz}$ and the temperature reaches $73.8 \mathrm{C}$, the temperature of the stator windings is 65.1 C, so it is necessary to take the insulation above Blevel.

(2) The calculation result of the casing surface temperature is $51.6^{\circ} \mathrm{C}$ and the actual measured result is $47.8^{\circ} \mathrm{C}$, which is due to the ignoring of the heat radiation.

(3) The casing temperature along the centerline of the movable tooth is influenced by the temperature conduction of casing at the location of the stator, so their temperature tends to be closer. 


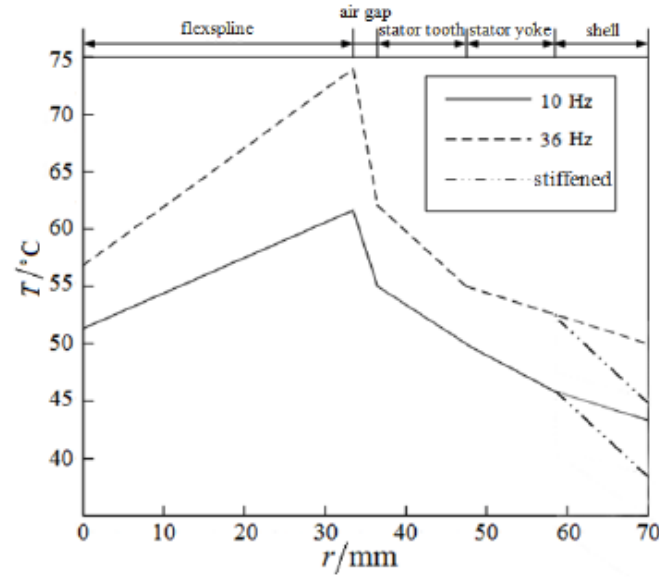

a) temperature distribution along the center line of the stator teeth

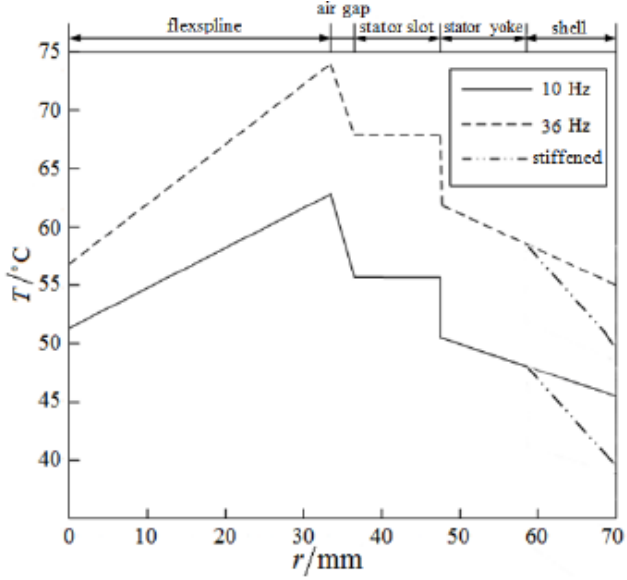

b) temperature distribution along the center line of the stator slot

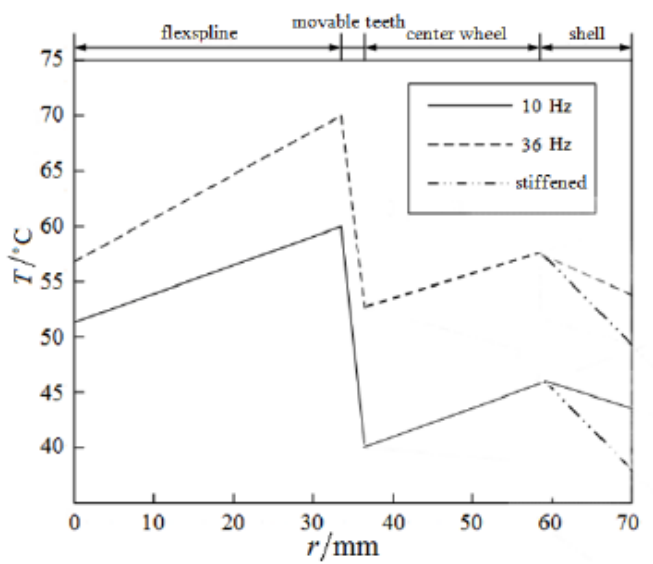

c) temperature distribution in the center line of the movable teeth

Fig. (2) Temperature distribution curve of the prototype.

(4) When the frequency increases, the system temperature will rise.

(5) The prototype surface is long cylindrical which is not conducive to heat dissipation; if the casing is ringstiffened, the calculated temperature can be reduced by $10^{\circ} \mathrm{C}$.

\section{CONCLUSION}

Based on the operating characteristics of electromagnetic harmonic tooth harmonic transmission system, this paper established the temperature field model and mathematical model for the system and gave out the calculation methods of the temperature filed the system, coupled with the test methods to verify the correctness of these methods. The results are shown as follows:

(1) In the process of solving, make appropriate assumptions to simplify the complex problems of three-dimensional temperature field to the two-dimensional one, which could reduce the workload. The two-dimensional finite element numerical method used to calculate the temperature of the system may have high accuracy.

(2) The temperature distribution of each part of the prototype along the radial direction is linear.
(3) As the system is a field with a variety of media, the temperature of different media may vary.

(4) The temperature of the system shows a linear upward trend with the increase of the load.

\section{CONFLICT OF INTEREST}

The authors confirm that this article content has no conflict of interest.

\section{ACKNOWLEDGEMENTS}

This work is supported by the National Natural Science Fund, China (No.E050203)

\section{REFERENCES}

[1] Delucchi M A, Jacobson M Z. "Providing all global energy with wind, water, and solar power, Part II: Reliability, system and transmission costs, and policies"[J]. Energy Policy, 2011, 39(3): 1170-1190.

[2] Li N, Wang S M, Du J J. "Natural characteristics and dynamic load coefficient of power four embranchments gear transmission"[J]. Journal of Aerospace Power, 2013, 28(2): 445-451.

[3] Ren Yubo, Xu Lizhong, Wu Yushuang. "Study on Principle of Electromechanical Integrated Electromagnetic Harmonic Oscillating Teeth Transmission" [J]. International Journal of Advancements in Computing Technology.2012. 4(16):183-192 
[4] Ren Yubo, Xu Lizhong. "Analysis on the Finite Element of Electromechanic Coupling of Flexible Gear in Electromagnetic Harmonic Drive". [J] Applied Mechanics and Mechanical Engineering. 2013. 249-250(15): 771-777.

[5] Ren Yubo,Xu Lizhong,Liang Yongli. "Analysis of flexible spline deformation in electromagnetic harmonic drive motor coupling system" [J]. Journal of Yanshan University. 2013, 3(37): 211-216

[6] Ren Yubo, Xu Lizhong,Liang Yongli. "Force and Deformation Analyses for Flexspline of an Electromechanical Integrated Electromagnetic Harmonic Friction Drive". ,China Mechanical Engineering.2011,22 (10) : 1169-1172

[7] Bracikowski N, Hecquet M, Brochet P V, et al. "Multiphysics modeling of a permanent magnet synchronous machine by using lumped models" [J] . IEEE Transactions on Industrial Electronics, 2012, 59 (6) : 2426-2437.

[8] Nerg J, Rilla Pyrhnen J. "Thermal analysis of radial-flux electrical machines with a high power density" $[\mathrm{J}]$. IEEE Transactions on Industrial Electronics, 2008， 55(10) : 3543-3554.

[9] Alberti L, Bianchi N. "A coupled thermal-electromagnetic analysis for a rapid and accurate prediction of IM performance" $[\mathrm{J}]$. IEEE Transactions on Industrial Electronics, 2008, 55 (10) : 3575-3582.

[10] Li weili, Yuan Shipeng, Huo Feiyang, et al. "Calculation of temperature field of PM generator for wind turbine based on theory of fluid heat transfer" $[\mathrm{J}]$. Electric Machines and Control, 2011, 15(9): $56-62$.

[11] Galea M, Gerada C, Raminosoa T. "A thermal improvement technique for the phase windings of electrical machines" $[\mathrm{J}]$. IEEE Transactions on Industry Applications, 2012, 48(1) : 79 87.

[12] Wen Jiabin, Wang Guohui. "Calculation and analysis of 3D size high voltage asynchronous temperature fields of medium motor based on coupled field" $[\mathrm{J}]$. Electric Machines and Control, 2011, 1(15): 74-78.

[13] Cheng Shu-kang, Li Cui-ping, Chai Feng. "Analysis of the 3D steady temperature field of induction motors with different cooling structures in mini electric vehicles" $[\mathrm{J}]$. Proceedings of the CSEE, 2012, 32(30): 82-90.

[14] Wang Yue-qing, Wu Gui-sheng, Wang Shi. Engineering electromagnetic fields introduction $[\mathrm{M}]$. Beijing: Electronic Industry Press, 2005: $198-210$

[15] Iwasaki S, Deodhar R. " Influence of PWM on the Proximity Loss in Permanent-Magnet Brushless AC Machines"[C]. IEEE Transactions on Industry Applications, 2009, 45(4): 1359-1367.

[16] Hu Min-qiang, Huang Xue-liang. Method and application of numerical calculation of motor performance [M]. Nanjing: Southeast University press, 2003: 72-77.

[17] LI Yourong, Wu Shuangying. Heat transfer theory [M]. Beijing: Science Press, 2012: 122-128.

Received: October 16, 2014

Revised: November 24, 2014

Accepted: November 28, 2014

(C) Yubo and Lizhong; Licensee Bentham Open.

This is an open access article licensed under the terms of the (https://creativecommons.org/licenses/by/4.0/legalcode), which permits unrestricted, noncommercial use, distribution and reproduction in any medium, provided the work is properly cited. 